\title{
Regional Climate Change: Downscaling, Prediction, and Impact Assessment
}

\author{
Lian Xie, ${ }^{1}$ Fredrick Semazzi, ${ }^{1}$ Adel Hanna, ${ }^{2}$ Richard Anyah, ${ }^{3}$ Huiwang Gao, ${ }^{4}$ and Yijun $\mathrm{He}^{5}$ \\ ${ }^{1}$ North Carolina State University, Raleigh, NC, USA \\ ${ }^{2}$ University of North Carolina at Chapel Hill, Chapel Hill, NC, USA \\ ${ }^{3}$ University of Connecticut, Storrs, CT, USA \\ ${ }^{4}$ Ocean University of China, Qingdao, China \\ ${ }^{5}$ Nanjing University of Information Science and Technology, Nanjing, Jiangsu, China
}

Correspondence should be addressed to Lian Xie; lian_xie@ncsu.edu

Received 5 January 2015; Accepted 5 January 2015

Copyright (C) 2015 Lian Xie et al. This is an open access article distributed under the Creative Commons Attribution License, which permits unrestricted use, distribution, and reproduction in any medium, provided the original work is properly cited.

Although the issue of climate change is often dealt with in global perspective, the impact of climate change must be assessed at regional scales. While global climate models can provide projections of the average state of large-scale circulation of future climate, the downscaling of such projections to regional scale with improved spatial and temporal resolution for both the forcing fields and the climatic responses is the basis for assessing the societal impacts of climate change. Therefore, it is important to not only study climate change at the global scale but also study the regional manifestations of the climate system at spatial scales ranging from less than a hundred kilometers to thousands of kilometers with time scales from months to years to decades.

This special issue publishes a collection of articles covering a wide range of topics of our understanding of "regional climate" from downscaling the variability of extreme rainfall over the Yangtze River basin (T. Gao and L. Xie) and assessing the water resources in the Yellow River region ( $\mathrm{Z}$. Wu et al.) in China to forecasting the precipitation and water resources in the Lake Victoria region in East Africa (X. Sun et al., R. Argent et al., and K. A. Smith and F. H. M. Semazzi), from downscaling wind energy resources in the contiguous United States (B. Liu et al.) to characterizing the precipitation extremes in the Carpathian region in central and southern Europe (L. Gaál et al.), and from analyzing the energy balance in semiarid grasslands in China (Q. Jiang et al.) to detecting future climate change signals in central and eastern Europe from numerical model simulations (M. Belda et al.). This special issue also includes articles addressing the impacts of regional climate change on tropical cyclones over the Atlantic Ocean (K. Xie and B. Liu), on crop yields in North China (H. Liu et al.), and on litter production and nutrient dynamics in a plantation in China (X. Ge et al.), as well as rainfall and drought in Eastern Kenya (M. O. Kisaka et al.). Additionally, several articles with focus on regional climate downscaling methodologies are also included. S. Kim et al. studied the effects of geographic features in a mountainous area on the downscaling of global climate model data; T. R. Lee et al. demonstrated the feasibility of using PRISM (parameterelevation regression on independent slope model) to downscale maximum temperature to subkilometer scale; L. Gao et al. applied the LASSO algorithm to statistically downscale the ERA-interim precipitation forecast over complex terrain; and K.-H. Min and W.-Y. Sun explored the application of an atmosphere-cryosphere coupled model in regional climate applications.

These articles reflect the recent advances and applications in "regional climate downscaling, prediction, and impact assessment" from a set of unique angles. We hope they are of interest to peers.

Lian Xie

Fredrick Semazzi

Adel Hanna

Richard Anyah

Huiwang Gao

Yijun $\mathrm{He}$ 

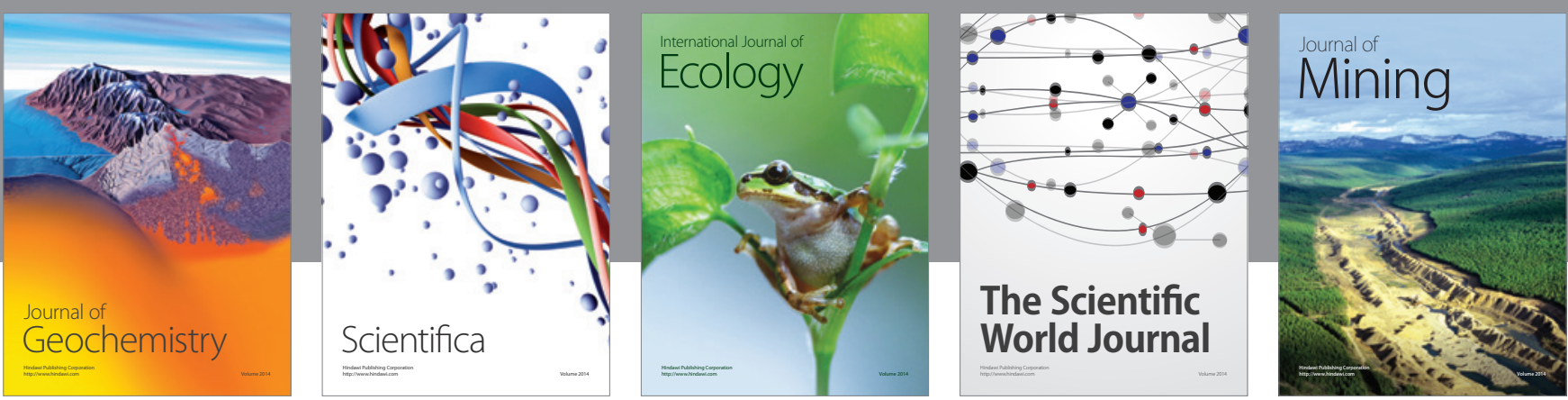

The Scientific World Journal
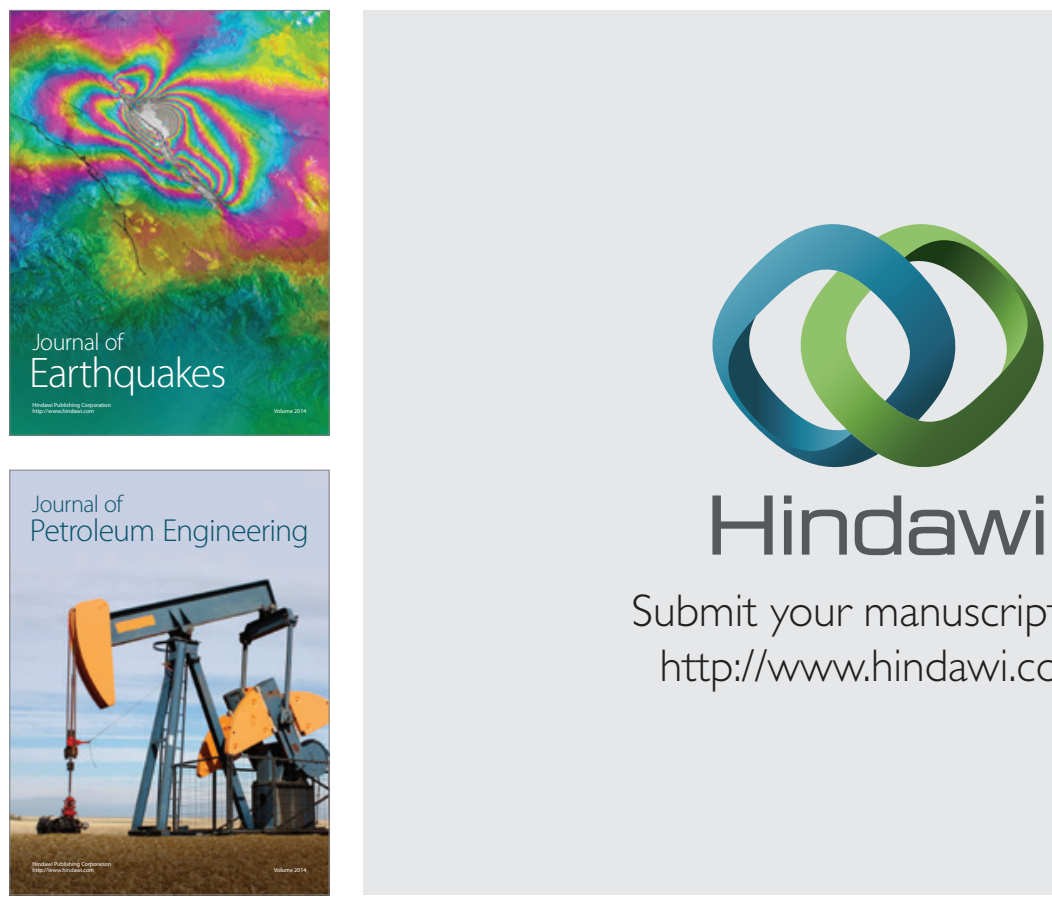

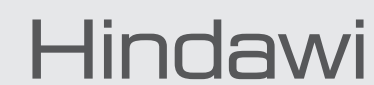

Submit your manuscripts at

http://www.hindawi.com
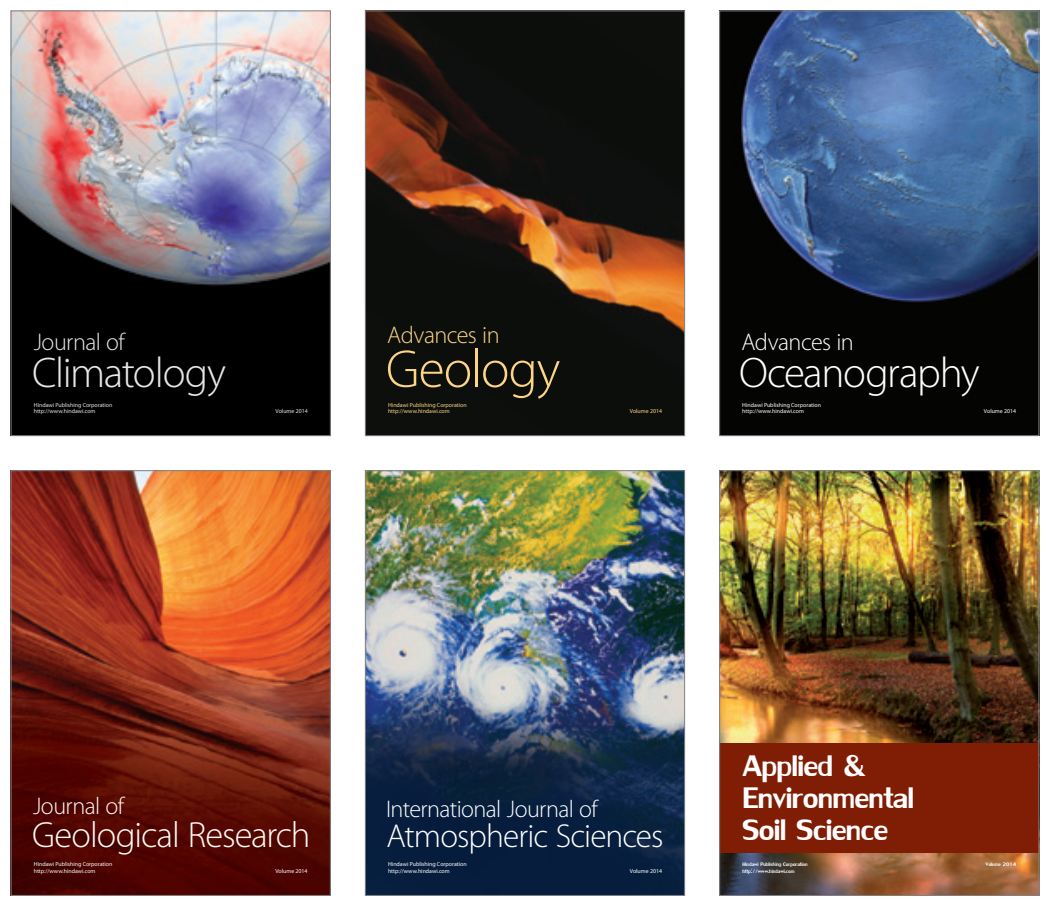
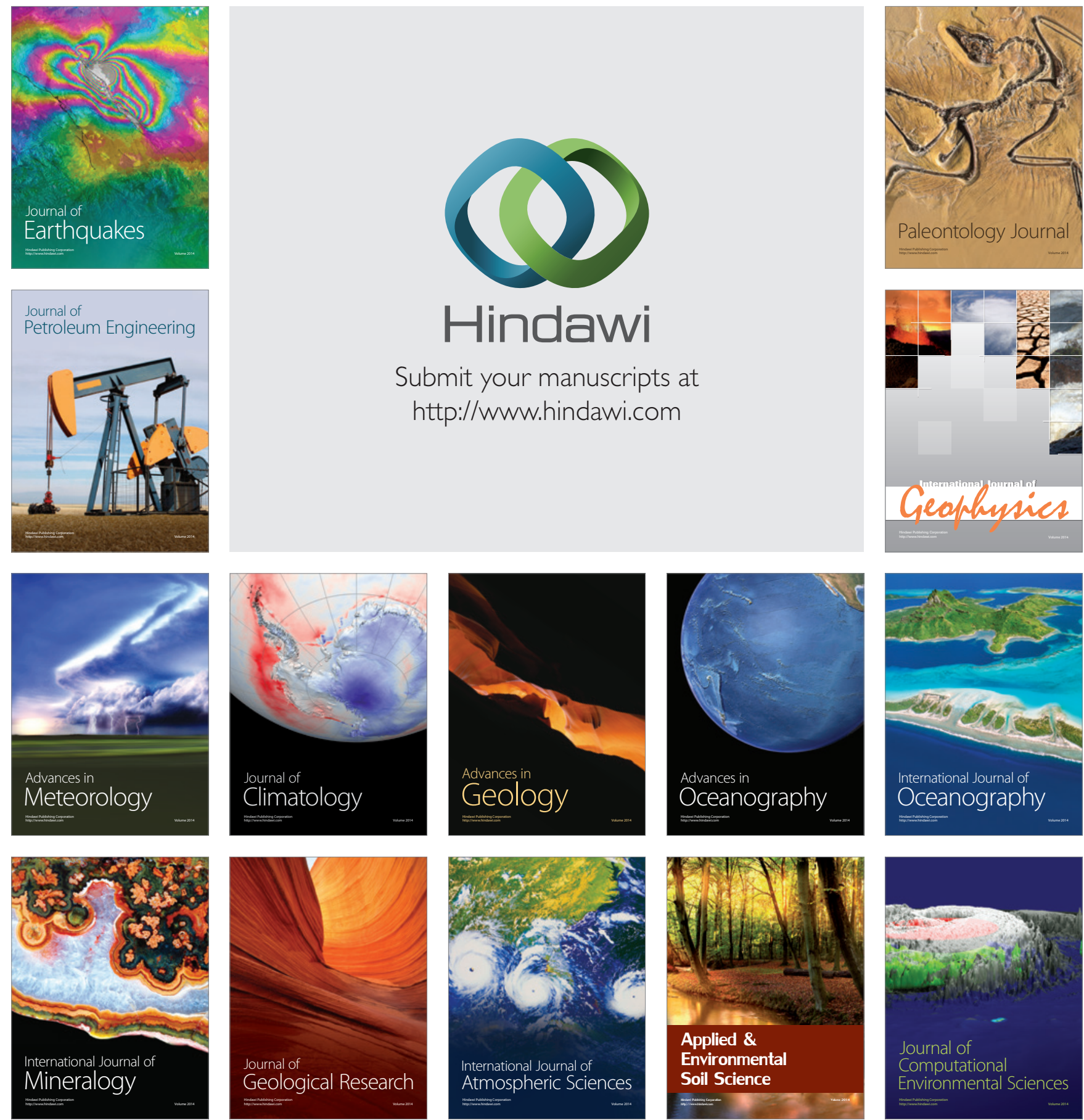